\title{
Genetic diversity and molecular analysis of metallo beta lactamases among imipenem resistant clinical isolates of Pseudomonas aeruginosa from Peshawar, Pakistan
}

\author{
Amjad Ali ${ }^{1}$, Kafeel Ahmad ${ }^{2}$, Shaista Rahat ${ }^{3}$, Israr Ahmad ${ }^{4}$
}

\begin{abstract}
Objectives: Pseudomonas aeruginosa is an opportunistic pathogen with remarkable adaptation ability to thrive in diverse environmental conditions. This study aimed at phenotypic and molecular analysis of metallo beta lactamases (blaIMP, blaVIM, blaNDM-1 and blaSPM-1) and genetic diversity analysis among imipenem resistant clinical isolates of Pseudomonas aeruginosa.

Methods: This study was conducted from May 2017 to June 2018. The study included 187 Pseudomonas aeruginosa isolates collected from different clinical specimens from Peshawar, Pakistan. The isolates were analyzed for resistance to imipenem. Combined disc test (CDT) was then performed for phenotypic detection of metallo beta lactamases among imipenem resistant isolates of Pseudomonas aeruginosa. Molecular detection of metallo beta lactamases genes i.e. blaIMP, blaVIM, blaNDM-1 and blaSPM-1 was analyzed through polymerase chain reaction. Genetic diversity was determined through RAPD-PCR.

Results: MBL production was observed in $76 \%(n=19)$ isolates. The occurrence of MBL genes blalMP, blaNDM-1 and blaVIM was $68 \%(n=17), 48 \%(n=12)$, and $4 \%(n=1)$ respectively. The blaSPM-1 gene was not detected. High genetic diversity was observed in current study. Out of 182 isolates 171 isolates showed different RAPD profiles (93.95\% polymorphism); 160 were unique RAPD strains and based on similarity coefficient $\geq$ $80 \%, 22$ isolates were clustered into 11 distinct clones.

Conclusion: A high prevalence of blalMP and blaNDM-1 among imipenem resistant isolates of Pseudomonas aeruginosa is alarming that calls for proper control and prevention strategies. RAPD technique was found to be a good genotyping technique when limited resources are available.
\end{abstract}

KEYWORDS: Pseudomonas aeruginosa, Genetic diversity, RAPD, MBL, blalMP, blaVIM, blaNDM-1.

doi: https://doi.org/10.12669/pjms.37.7.4303

How to cite this:

Ali A, Ahmad K, Rahat S, Ahmad I. Genetic diversity and molecular analysis of metallo beta lactamases among imipenem resistant clinical isolates of Pseudomonas aeruginosa from Peshawar, Pakistan. Pak J Med Sci. 2021;37(7):1865-1870. doi: https://doi.org/10.12669/pjms.37.7.4303

This is an Open Access article distributed under the terms of the Creative Commons Attribution License (http://creativecommons.org/licenses/by/3.0), which permits unrestricted use, distribution, and reproduction in any medium, provided the original work is properly cited.

. Amjad Ali, PhD.

2. Kafeel Ahmad, PhD.

3. Shaista Rahat, PhD Scholar.

4. Israr Ahmad, PhD.

Pakistan Health Research Council,

Khyber Medical College, Peshawar, Pakistan.

1-3: Center of Biotechnology and Microbiology,

University of Peshawar, Peshawar, Pakistan.

Correspondence:

Dr. Kafeel Ahmad, PhD,

Assistant Professor, Center of Biotechnology and Microbiology, University of Peshawar, Post Code: 25200,

Peshawar, Pakistan.

E-mail: kafeelpbg@gmail.com

* Received for Publication:

* Revision Received:

* Revision Accepted:

February 19, 2021

June 25, 2021

July 18,2021

\section{INTRODUCTION}

Extensive drug resistant strains of Pseudomonas aeruginosa have been reported from hospitals around the world. ${ }^{1}$ Resistance of Pseudomonas aeruginosa to different classes of antibiotics such as penicillin, cephalosporin, quinolone, aminoglycoside and carbapenem has been demonstrated. ${ }^{2}$ Metallo beta lactamases (MBL) are enzymes that catalyze the hydrolysis of broad-spectrum beta lactam antibiotics including carbapenems. ${ }^{3}$ Metallo beta lactamases (MBL) production has been reported from Brazil, Iran and India among carbapenem resistant clinical isolates of Pseudomonas aeruginosa. ${ }^{4-6}$ Among beta lactam antibiotics, carbapenems are the most 
effective against Gram negative and Gram-positive bacteria demonstrating broad range antibacterial activity. ${ }^{7}$ Resistant to carbapenems especially in Gram negative pathogens is global public health issue because of the spread of transferable carbapenemase encoding genes. ${ }^{7}$ Highest resistance was reported by Farooq et al., against imipenem in multi drug resistant clinical isolates of Pseudomonas aeruginosa from tertiary care hospital Karachi, Pakistan. ${ }^{8}$ Imipenem resistant pathogenic bacterial strains have been reported from Lahore and Quetta Pakistan., ${ }^{910}$ Imipenemase (IMP), Verona integron encoded metallo beta lactamase (VIM), New Delhi metallo beta lactamase (NDM), Sao Paulo metallo beta lactamase (SPM), Florence imipenemase (FIM) and Germany imipenemase (GIM) are various types of metallo beta lactamases that have been reported in carbapenem resistant isolates of Pseudomonas aeruginosa. ${ }^{11}$ Genes for MBLs are located on integrons, plasmids, transposons or on chromosomes. ${ }^{11}$ These specific genetic elements carry carbapenem and other antibiotics resistant determinants and thus confer multi drug resistance to Pseudomonas aeruginosa. These antibiotic resistant elements could be transferred to other Gram-negative bacterial strains and contribute towards spread of antimicrobial resistance rate making the treatment of infected patients complex. ${ }^{11}$ It is therefore, important to know about the epidemiology and resistance mechanisms of Pseudomonas aeruginosa in order to control and prevent multi drug resistant pathogenic strains to overcome possible health risks. ${ }^{11}$

Microbial typing is vital in order to determine the relationship between microbes. ${ }^{12}$ Knowledge about clonal relatedness between microbial strains is important to determine source, route of infection, detect cross transmission and confirm epidemic of pathogen. ${ }^{12}$ Microbial typing is also used in the recognition of virulent strains and to assess the effectiveness of control measures. Randomly amplified polymorphic DNA (RAPD) RAPD is a polymerase chains reaction (PCR) based genotyping method that requires short single arbitrary primers that could randomly amplify DNA and generate numerous discrete segments of DNA. ${ }^{13}$ RAPD demonstrates quick and efficient DNA polymorphism at large number of loci in genome of an organism. RAPD is simple, efficient, low-cost technique that needs no prior knowledge of target DNA sequence. ${ }^{13}$

No information is available on the occurrence of metallo beta lactamases and genetic diversity of Pseudomonas aeruginosa from Pakistan. Hence, this study aimed at analysis of MBL genes and genetic diversity of Pseudomonas aeruginosa isolates from the region.

\section{METHODS}

Bacterial isolates and identification: A total of 187 clinical isolates of Pseudomonas aeruginosa were collected from tertiary care hospitals (Khyber Teaching Hospital (KTH) and Hayatabad Medical Complex (HMC)) of Peshawar, Pakistan in 2017-2018. These included 74 isolates from pus, 34 isolates from urine, 24 isolates from sputum, 21 isolates from wound, 12 isolates from bronchial wash, 8 isolates from cerebrospinal fluid, six isolates from blood, 5 isolates from high vaginal swab and 3 isolates from diabetic foot. Patient's informed consents and permission were obtained from the participating hospitals and study was ethically approved by Post Graduate Medical Institute (PGMI) Peshawar, KP Pakistan (Ref: 1046/PGMI, Dated: 03-05-2017). Cultures were inoculated on MacConkey agar (Oxoid, UK) and were incubated for 24 hours at $37^{\circ} \mathrm{C}$. Using standard procedures pure isolates were identified as Pseudomonas aeruginosa through morphological (Gram staining) and biochemical tests (catalase, oxidase, citrate utilization test, triple sugar iron test, indole test and nitrate reduction test). ${ }^{14}$

Phenotypic detection of Metallo Beta Lactamases $(M B L)$ : Twenty-five imipenem resistant isolates among the 187 isolates were subjected to phenotypic and genotypic detection of metalo lactamases. Combine disc test (CDT) was performed for phenotypic detection of MBL genes. Two imipenem discs (10 $\mu \mathrm{g}$; Oxoid, UK) were placed $25 \mathrm{~mm}$ to 30 $\mathrm{mm}$ apart on surface of Muller Hinton agar (Oxoid, UK) inoculated with Pseudomonas aeruginosa. One imipenem disc was impregnated with $5 \mu$ EDTA (0.5M, pH 8.0). Culture plates were incubated for 18 to 24 hours at $37^{\circ} \mathrm{C}$. Zones of inhibition were compared. The bacterial strain was considered as MBL positive if zone of inhibition of imipenemEDTA disc was $\geq 7 \mathrm{~mm}$ than the zone of imipenem disc alone. ${ }^{5}$

DNA extraction: GeneJET Genomic DNA purification kit (Thermo Scientific, Lithuania Cat. No. K0721) was used for DNA extraction. Recommended protocol was followed, and the quality of DNA was confirmed through gel electrophoresis using $0.8 \%$ agarose gel. The extracted DNA was preserved at $-20^{\circ} \mathrm{C}$ for further use.

Molecular detection of MBLs: Four metalo beta lactamases genes i.e., blaIMP, blaVIM, blaNDM-1 and blaSPM-1 were analyzed among 25 imipenem 
resistant isolates of Pseudomonas aeruginosa using previously reported primers and amplification profiles. ${ }^{15-17}$ The amplified PCR products were analyzed through gel electrophoresis and 100 base pair DNA ladder (Bioron, Cat. No. 304105) was used as size marker.

RAPD-PCR: Genetic diversity was determined through RAPD-PCR using previously reported primer (RAPD 272) and procedures. ${ }^{18}$ Amplified products were analyzed through gel electrophoresis. The sizes of amplified DNA fragments were compared with 100 base pair (bp) DNA ladder (Bioron, Cat. No. 304105). RAPD PCR was repeated multiple times to ensure the reproducibility of the results and overcome the limitations of RAPD technique in a low-income setting.
Statistical Analysis: Amplified bands through RAPD-PCR were scored as absent or present to generate bivariate (0-1) data for statistical analysis. Genetic diversity among the clinical isolates of Pseudomonas aeruginosa was determined using this bivariate data. Genetic distances among isolates were calculated using Nei and Li method by the formula $\mathrm{D}_{\mathrm{xy}}=1-\left(\mathrm{N}_{\mathrm{xy}}\right) /\left(\mathrm{N}_{\mathrm{x}}+\mathrm{N}_{\mathrm{y}}-\mathrm{N}_{\mathrm{xy}}\right)$ as reported. ${ }^{19}$ The term $\mathrm{D}_{\mathrm{xy}}$ represents dissimilarity distance between two genotypes $X$ and $Y$. Term $N_{x y}$ represent the number of common bands present between two genotypes. The term $\mathrm{N}_{\mathrm{x}}$ represent total number of bands present in genotype $X$ and $N_{y}$ shows total number of bands present in genotype Y. Using MEGA 7 (Molecular Evolutionary Genetics Analysis, version 7) software based

Table-I: Comparison of phenotypic MBL detection and occurrence of MBL genes (blaIMP, blaNDM-1, blaVIM and blaSPM-1) among imipenem resistant clinical isolates of Pseudomonas aeruginosa (+ means presence, - means absence).

\begin{tabular}{|c|c|c|c|c|c|c|c|}
\hline \multirow{2}{*}{ S. No } & \multirow{2}{*}{ Isolate } & \multirow{2}{*}{ Specimen } & \multirow{2}{*}{$\begin{array}{c}\text { Phenotypic } M B L \\
\text { status }\end{array}$} & \multicolumn{4}{|c|}{ Occurrence of MBL genes } \\
\hline & & & & blaIMP & blaNDM-1 & blaVIM & blaSPM-1 \\
\hline 1 & R9 & Pus & - & - & - & - & - \\
\hline 2 & R20 & Blood & + & + & - & - & - \\
\hline 3 & R31 & Wound & + & - & + & - & - \\
\hline 4 & R55 & Wound & - & - & - & - & - \\
\hline 5 & R59 & Pus & + & + & + & - & - \\
\hline 6 & R60 & Bronchial Wash & + & + & + & - & - \\
\hline 7 & R61 & Wound & + & + & - & + & - \\
\hline 8 & R62 & Pus & + & + & + & - & - \\
\hline 9 & R63 & Urine & + & + & - & - & - \\
\hline 10 & R64 & Sputum & + & + & - & - & - \\
\hline 11 & N27 & Bronchial wash & + & + & + & - & - \\
\hline 12 & N29 & Bronchial wash & + & + & + & - & - \\
\hline 13 & N35 & Sputum & + & + & + & - & - \\
\hline 14 & N39 & Bronchial wash & + & + & + & - & - \\
\hline 15 & N40 & Urine & + & + & - & - & - \\
\hline 16 & N55 & Urine & + & + & + & - & - \\
\hline 17 & N69 & Sputum & - & - & - & - & - \\
\hline 18 & N91 & Wound & - & - & - & - & - \\
\hline 19 & N95 & Wound & - & - & - & - & - \\
\hline 20 & N100 & Urine & + & + & + & - & - \\
\hline 21 & N102 & Sputum & - & - & - & - & - \\
\hline 22 & H38 & Pus & + & + & - & - & - \\
\hline 23 & H65 & Urine & + & - & + & - & - \\
\hline 24 & M26 & Pus & + & + & - & - & - \\
\hline 25 & K31 & Pus & + & + & + & - & - \\
\hline
\end{tabular}




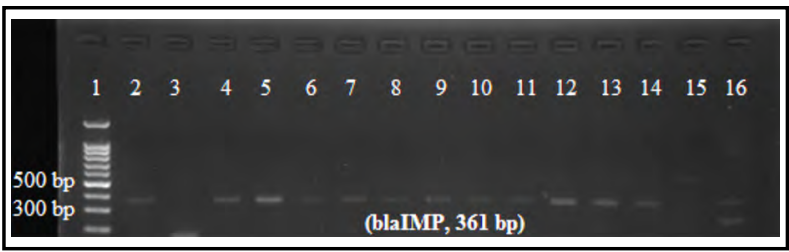

Fig.1: PCR amplification of blaIMP gene: Lane 1 (100 bp

DNA ladder); Lanes 2, 4, 5, 6, 7, 8, 9, 10, 11, 12, 13,

14 and 16 are blaIMP positive samples with

a 361 bp gene specific amplified fragment.

on UPGMA (Unweighted Pair Group Method with Arithmetic mean) method dendrogram was generated from bivariate data obtained from RAPD analysis. Dendrogram was further analyzed for clonal relatedness among the clinical isolates of Pseudomonas aeruginosa. Distinct clones among the clinical isolates were defined based on similarity coefficient $\geq 80 \% .^{20}$

\section{RESULTS}

Of 187 isolates of Pseudomonas aeruginosa, 25 $(13.36 \%)$ isolates were resistant to imipenem. Among these 25 imipenem resistant isolates, 19 $(76 \%)$ isolates were phenotypically MBL producers (Table-I). On the basis of phenotypic tests, frequency of MBL producing isolates of Pseudomonas aeruginosa among different clinical specimens was: blood $5.26 \%(\mathrm{n}=1)$, sputum $10.52 \%(\mathrm{n}=2)$, wound $10.52 \%$ $(n=2)$, bronchial wash $21.05 \%(n=4)$, urine $26.31 \%$ $(n=5)$ and pus $26.31 \%(n=5)$ as given in Table-I. Metallo beta lactamases genes blaIMP, blaNDM-1 and blaVIM were detected in $68 \%(\mathrm{n}=17), 48 \%$ $(\mathrm{n}=12)$ and $4 \%(\mathrm{n}=1)$ isolates respectively (Fig.13, Table-I). MBL gene blaSPM-1 was not detected. The occurrence of Metallo beta lactamases genes correlated well with phenotypic detection of MBLs (Table-I).

Electrophoresis pattern of RAPD PCR products revealed a total of 31 distinct bands ranging in size from 150-1600 bp (Fig.4). Genetic dissimilarity distance values ranged from 0-1. Dendrogram of

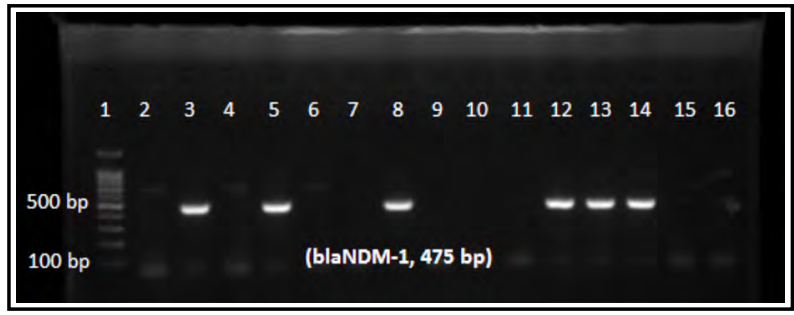

Fig.2: PCR amplification of blaNDM-1 gene: Lane 1 (100 bp DNA ladder); Lanes 3, 5, 8, 12, 13 and 14 contain blaNDM-1 (475 bp amplified fragment) positive samples of Pseudomonas aeruginosa.

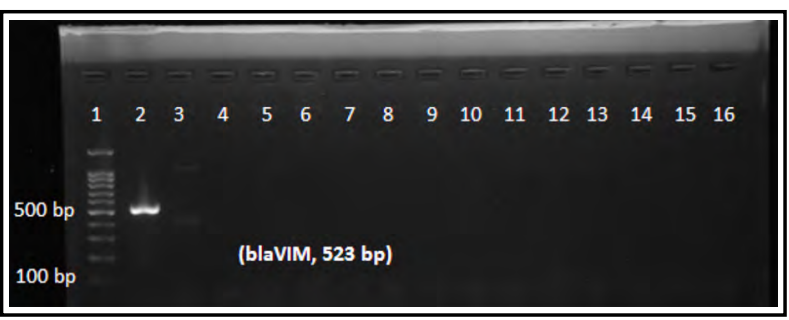

Fig.3: PCR amplification of blaVIM gene: Lane 1 (100 bp DNA ladder); Lane 2 contain blaVIM (523 bp amplified fragment) positive sample of Pseudomonas aeruginosa.

RAPD data is shown in Fig.5. Of the 182 isolates of Pseudomonas aeruginosa 171 isolates showed different RAPD profiles; 160 were unique RAPD strains and 22 isolates were clustered into 11 distinct clones. Genetic polymorphism was found to be $93.9 \%$. Distinct clones among the isolates were defined based on similarity coefficient $\geq 80 \%$. The 11 distinct clones were: R28, M36; M7, R55; H83, H23; H64, K25; N16, N17; N48, B2; N66, N68; N71, N90; H99, N23; M11, N32; R16, H62.

\section{DISCUSSION}

In the current study, a high prevalence $(76 \%)$ of MBLs was observed among imipenem resistant clinical isolates of Pseudomonas aeruginosa. Reports on MBL production in carbapenem resistant Pseudomonas aeruginosa have been reported from India $(16.87 \%)$, Brazil (16.1\%), Iran (60.4\%) and Korea $(41 \%))^{4,21-23}$ Patients infected with metallo beta lactamases producing bacteria are at escalated risk of treatment failure. ${ }^{24}$ Current study reported high prevalence of blaIMP (68\%) as compared to previous findings from Iraq (50\%), India (9\%) and Egypt $(4 \%)$ in carbapenem resistant isolates of

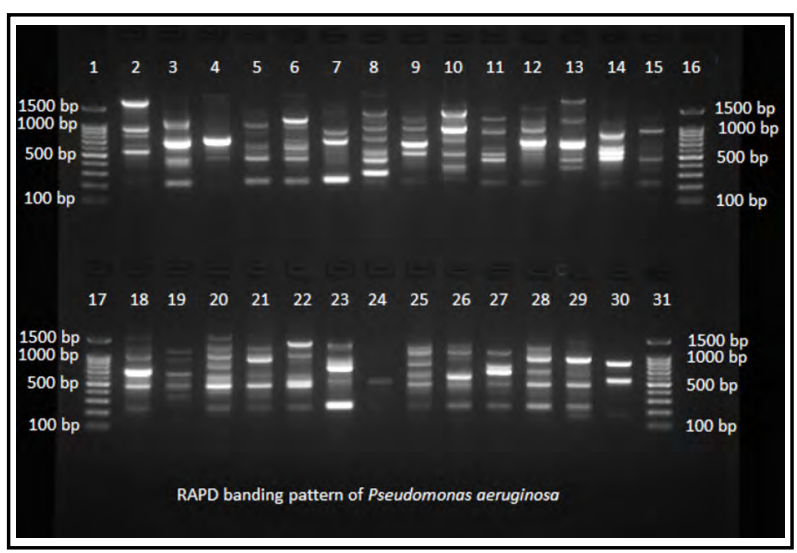

Fig.4: RAPD banding profile of Pseudomonas aeruginosa with RAPD primer 272: Lanes 1, 16, 17 and $31=100 \mathrm{bp}$ DNA ladder; Lanes 2, 3, 4, 5, 6, 7, 8, 9, 10, 11, 12, 13, 14, 15, 18, 19, 20, 21, 22, 23, 24, 25, 26, 27, 28, 29 and $30=$ RAPD banding pattern of Pseudomonas aeruginosa isolates. 


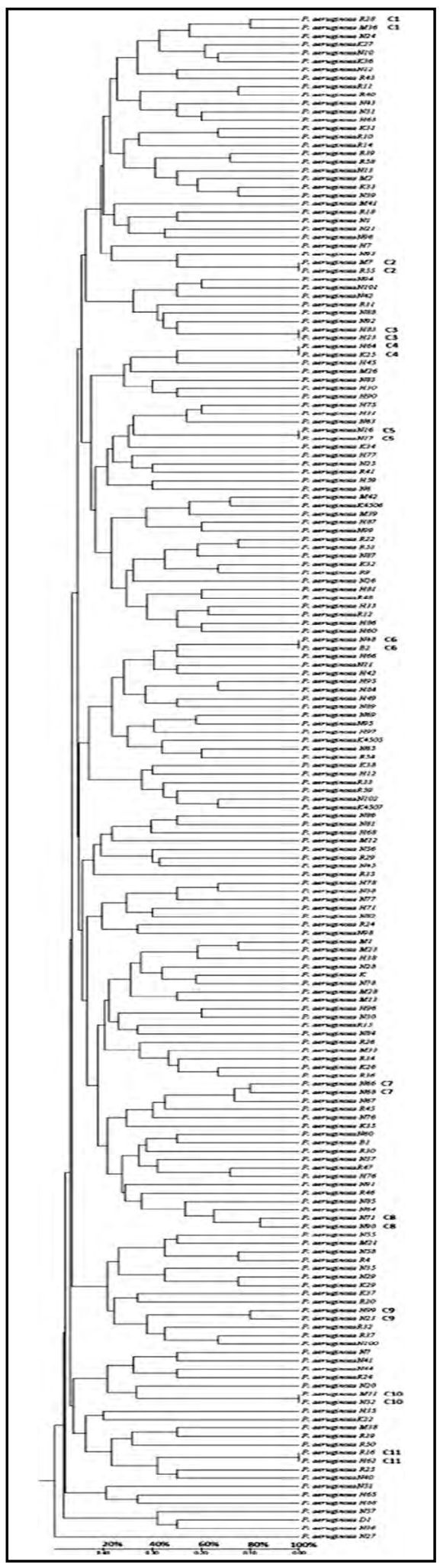

Fig.5: Dendrogram: Dendrogram generated using UPGMA method. Based on similarity coefficient of $\geq 80 \%, 11$ clones were distinguished as $\mathrm{C} 1=(\mathrm{R} 28, \mathrm{M} 36), \mathrm{C} 2=(\mathrm{M} 7, \mathrm{R} 55), \mathrm{C} 3=(\mathrm{H} 83, \mathrm{H} 23), \mathrm{C} 4=(\mathrm{H} 64$, $\mathrm{K} 25), \mathrm{C} 5=(\mathrm{N} 16, \mathrm{~N} 17), \mathrm{C} 6=(\mathrm{N} 48, \mathrm{~B} 2), \mathrm{C} 7=(\mathrm{N} 66, \mathrm{~N} 68), \mathrm{C} 8=(\mathrm{N} 71$, N90), $\mathrm{C} 9=(\mathrm{H} 99, \mathrm{~N} 23), \mathrm{C} 10=(\mathrm{M} 11, \mathrm{~N} 32) \& \mathrm{C} 11=(\mathrm{R} 16, \mathrm{H} 62)$.
Pseudomonas aeruginosa. ${ }^{25-27}$ High prevalence of bla-NDM-1 (48\%) was observed in current study in contrast to a previous report from India $(10 \%) .{ }^{26} \mathrm{Low}$ prevalence (4\%) of blaVIM in this study is in contrast to previous findings by Mohanam et al. from India with $32 \%$ blaVIM prevalence and Hashem et al. from Egypt with $20 \%$ blaVIM prevalence. ${ }^{26,27}$ BlaSPM-1 was not detected in current study. The gene has been reported previously from Iraq $(16.6 \%)$ and Egypt $(24 \%)$ in carbapenem resistant isolates of Pseudomonas aeruginosa. ${ }^{25,27}$ Rapid dissemination and increase in number of MBL producing Pseudomonas aeruginosa is worrisome and worldwide health problem.

Current study showed high genetic diversity (93.9\% polymorphism) between Pseudomonas aeruginosa isolates. A study by Silva et al. also revealed high genetic diversity (89.6\%) among 96 clinical isolates of Pseudomonas aeruginosa from Brazilian hospitals. ${ }^{18}$ Thirty different genotypes with $85.7 \%$ polymorphism were observed among Pseudomonas aeruginosa strains recovered from cystic fibrosis patients in Canada. ${ }^{28}$ In contrast to current study, a low genetic diversity $(18 \%)$ among 50 isolates of Pseudomonas aeruginosa from burn patients was reported from Iran. ${ }^{29}$ This low level of genetic diversity was attributed to cross infection of bacteria among burns patients within the same hospital. Epidemiologically, genetically relatedness of bacterial isolates suggests origin and spread from a common source. The high level of genetic diversity polymorphism in current study could be attributed to location of isolation and diverse clinical specimens used for isolation.

Limitations of the study: Current study involved sampling for hospitals of Peshawar. A large sampling area could help in better understanding of the epidemiological aspects of resistant strains of Pseudomonas aeruginosa at molecular level on regional level.

\section{CONCLUSION}

The high prevalence of metallo beta lactamases genes blaIMP, blaNDM-1 and blaVIM among imipenem resistant strains of Pseudomonas aeruginosa is a serious health concern. Strict hygienic conditions, good infection control policies and appropriate use of antibiotics are essential to prevent further dissemination of MBL producing strains of Pseudomonas aeruginosa. It is also concluded that genotypic surveillance study must be performed on regular basis to identify possible sources of dissem- 
ination and origin of pathogenic bacteria. In this regard, RAPD technique offers low cost and quick method especially in developing countries.

Grant Support: Higher Education Commission (HEC) Islamabad, Pakistan under the Indigenous PhD scholarship scheme.

\section{Conflict of interest: None.}

\section{REFERENCES}

1. Buhl M, Peter S, Willmann M. Prevalence and risk factors associated with colonization and infection of extensively drug-resistant Pseudomonas aeruginosa: A systematic review. Expert Rev Anti Infect Ther. 2015;13(9):1159-1170. doi: 10.1586/14787210.2015.1064310

2. Lambert PA. Mechanisms of antibiotic resistance in Pseudomonas aeruginosa. J R Soc Med. 2002;95(Suppl-41):22-26.

3. Palzkill T. Metallo- $\beta$-lactamase structure and function. Ann N Y Acad Sci. 2013;1277:91-104. doi: 10.1111/j.17496632.2012.06796.x

4. Gonçalves IR, Dantas RCC, Ferreira ML, Batistao DWDF, Gontijo-Filho PP, Ribas RM. Carbapenem-resistant Pseudomonas aeruginosa: association with virulence genes and biofilm formation. Braz J Microbiol. 2017;48(2):211-217. doi: 10.1016/j. bjm.2016.11.004

5. Moosavian M, Rahimzadeh M. Molecular detection of metallo$\beta$-lactamase genes, blaIMP-1, blaVIM-2 and blaSPM- 1 in imipenem resistant Pseudomonas aeruginosa isolated from clinical specimens in teaching hospitals of Ahvaz, Iran. Iran J Microbiol. 2015;7(1):2-6.

6. Mishra SN, Biswal SR, Behera BK, Pattnaik D. Detection of prevalence of metallo-beta lactamases in clinical isolates of imipenem resistant Pseudomonas aeruginosa from neonatal septicaemia cases in a tertiary hospital in Odisha, India. Int J Contemp Pediatr. 2018;5(1):61-66. doi: 10.18203/2349-3291.ijcp20175540

7. Meletis G. Carbapenem resistance: overview of the problem and future perspectives. Ther Adv Infect Dis. 2016;3(1):15-21. doi: $10.1177 / 2049936115621709$

8. Farooq L, Memon Z, Ismail MO, Sadiq S. Frequency and antibiogram of multi-drug resistant pseudomonas aeruginosa in a Tertiary Care Hospital of Pakistan. Pak J Med Sci. 2019;35(6):1622-1626. doi: 10.12669/pjms.35.6.930

9. Nosheen S, Bukhari NI, Ejaz H, Abbas N. Antibiogram and recent incidence of multi-drug resistant carbapenemase producing Escherichia coli isolated from paediatric patients. Pak J Med Sci. 2020;36(2):246-250. doi: 10.12669/pjms.36.2.928

10. Pirkani GS, Awan MA, Abbas F, Din M. Culture and PCR based detection of bacteria causing urinary tract infection in urine specimen. Pak J Med Sci. 2020;36(3):391-395. doi: $10.12669 /$ pjms.36.3.1577

11. Hong DJ, Bae IK, Jang IH, Jeong SH, Kang HK, Lee K. Epidemiology and characteristics of metallo- $\beta$-lactamase producing Pseudomonas aeruginosa. Infect Chemother. 2015;47(2):81-97. doi: $10.3947 /$ ic.2015.47.2.81

12. Ranjbar R, Karami A, Farshad S, Giammanco GM, Mammina C. Typing methods used in the molecular epidemiology of microbial pathogens: a how-to guide. New Microbiol. 2014;37(1):1-15.

13. Kumari N, Thakur SK. Randomly amplified polymorphic DNA-a brief review. Am J Anim Vet Sci. 2014;9(1):6-13. doi: 10.3844/ajavsp.2014.6.13

14. Parija SC. Textbook of Practical Microbiology. India: Ahuja Publisher, 2006.

15. Peerayeh SN, Mahabadi RP, Toupkanlou SP, Siadat SD. Diversity of $\beta$-lactamases produced by imipenem resistant, Pseudomonas aeruginosa isolates from the bloodstream. Burns. 2014;40(7):1360-1364. doi: 10.1016/j.burns.2014.01.009
16. Shanthi M, Sekar U, Kamalanathan A, Sekar B. Detection of New Delhi metallo beta lactamase-1 (NDM-1) carbapenemase in Pseudomonas aeruginosa in a single centre in southern India. Indian J Med Res. 2014;140(4):546-550.

17. Franco MRG, Caiaffa-Filho $\mathrm{HH}$, Burattini MN, Rossi $\mathrm{F}$. Metallo-beta-lactamases among imipenem-resistant Pseudomonas aeruginosa in a Brazilian university hospital. Clinics. 2010;65(9):825-829. doi: 10.1590/s1807-59322010000900002

18. Silva LV, Galdino ACM, Nunes APF, dos Santos KR, Moreira BM, Cacci LC, et al. Virulence attributes in Brazilian clinical isolates of Pseudomonas aeruginosa. Int J Med Microbiol. 2014;304(8):990-1000. doi: 10.1016/j.ijmm.2014.07.001

19. Nei M, Li WH. Mathematical model for studying genetic variation in terms of restriction endonucleases. Proc Natl Acad Sci. 1979;76(10):5269-5273. doi: 10.1073/pnas.76.10.5269

20. Waters V, Zlosnik JEA, Yau YCW, Speert DP, Aaron SD, Guttman DS. Comparison of three typing methods for Pseudomonas aeruginosa isolates from patients with cystic fibrosis. Eur J Clin Microbiol Infect Dis. 2012;31(12):3341-3350. doi: 10.1007/ s10096-012-1701-z

21. Tankhiwale S. Beta-lactamases in P. aeruginosa: a threat to clinical therapeutics. Curr Pediatr Res. 2016;20(1\&2):253-257.

22. Sheikh AF, Rostami S, Jolodar A, Tabatabaiefar MA, Khorvash F, Saki A, et al. Detection of metallo-beta lactamases among carbapenem-resistant Pseudomonas aeruginosa. Jundishapur J Microbiol. 2014;7(11):e12289. doi: 10.5812/jjm.12289

23. Cho HH, Kwon GC, Kim S, Koo SH. Distribution of pseudomonas-derived Cephalosporinase and Metallo- $\beta$-lactamases in Carbapenem-resistant Pseudomonas Aeruginosa isolates from Korea. J Microbiol Biotechnol. 2015;25(7):1154-1162. doi: $10.4014 / \mathrm{jmb} .1503 .03065$

24. Khorvash F, Yazdani M, Shabani S, Soudi A. Pseudomonas aeruginosa-producing metallo- $\beta$-lactamases (VIM, IMP, SME, and AIM) in the clinical isolates of intensive care units, a university hospital in Isfahan, Iran. Adv Biomed Res. 2017;6(147):1-6. doi: 10.4103/2277-9175.219412

25. Al-Charrakh AH, Al-Awadi SJ, Mohammed AS. Detection of metallo- $\beta$-lactamase producing Pseudomonas aeruginosa isolated from public and private hospitals in Baghdad, Iraq. Acta Medica Iranica. 2016;54(2):107-113.

26. Mohanam L, Menon T. Coexistence of metallo-beta-lactamaseencoding genes in Pseudomonas aeruginosa. Indian J Med Res. 2017;146(Suppl-1):S46-S52. doi: 10.4103/ijmr.IJMR_29_16

27. Hashem H, Hanora A, Abdalla S, Shaeky A, Saad A. Dissemination of metallo- $\beta$-lactamase in Pseudomonas aeruginosa isolates in Egypt: mutation in bla VIM-4. APMIS. 2017;125(5):499-505. doi: 10.1111/apm.12669

28. Hafiane A, Ravaoarinoro M. Characterization of Pseudomonas aeruginosa strains isolated from cystic fibrosis patients by different typing methods. Pathol Biol. 2011;59(5):e109-e114. doi: 10.1016/j.patbio.2009.06.002

29. Nanvazadeh F, Khosravi AD, Zolfaghari MR, Parhizgari N. Genotyping of Pseudomonas aeruginosa strains isolated from burn patients by RAPD-PCR. Burns. 2013;39(7):1409-1413. doi: 10.1016/j.burns.2013.03.008

\section{Author's Contribution:}

AA: Did samples collection, conducted experiments and wrote manuscript and responsible and accountable for the accuracy and integrity of the work. KA: Designed and supervised the study, analyzed the data and proofread the manuscript. SR: Helped in literature search, cooperated during experiments and data interpretation. IA: Performed data analysis and proof read the manuscript. 